\title{
TRACKING OF ELECTRON BEAMS WITH NUMERICALLY DETERMINED SPACE CHARGE FORCES*
}

\author{
J. Staats $^{\#}$, T. Weiland, Theorie Elektromagnetischer Felder, TU Darmstadt; \\ S. Kostial, A. Richter, Institut für Kernphysik, TU Darmstadt
}

\begin{abstract}
A tracking algorithm for the description of electron beams using grid-based space charge fields is compared to a method based on point-to-point calculations. The charged particles' equations of motion are solved with a fifth-order embedded Runge-Kutta method using the concept of macroparticles. The space charge forces are determined in the bunch's restframe with a multigrid-method [1].
\end{abstract}

\section{INTRODUCTION}

In cases of space charge fields being approximately piecewise constant, the space charge fields can be determined in the beam's rest frame. Using a grid-based method for calculating the electrostatic potential $\varphi^{\prime}$ within the beam has advantages with respect to particle statistics. The electric field of a relativistic electron with velocity $v$ in the laboratory system is focused in transverse direction. The space angle enclosing a certain share of the electron's electric flux (field cone) thus has a decreasing transverse opening angle if the particle's energy increases. The transversal dominated space charge fields of a $10 \mathrm{MeV}$ electron beam demonstrate the asymmetry due to relativistics (see Fig. 1).

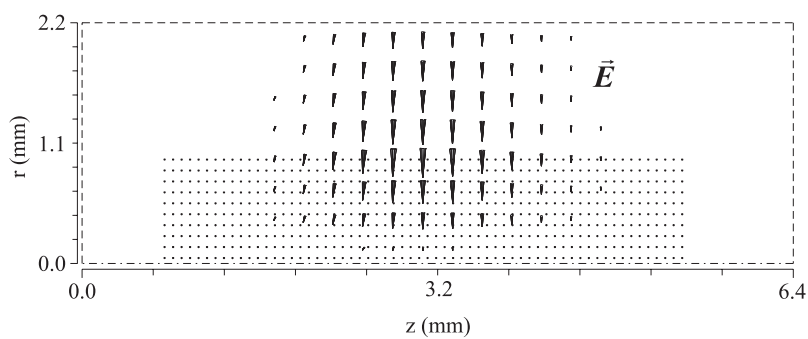

Figure 1: Electric space charge fields of a cylindrical Gaussian electron beam, $10 \mathrm{MeV}$, calculated with MAFIA TS2 code.

Calculating space charge forces by summation over all point-to-point interactions one has to use enough particles in order to keep the statistical fluctuation of the number of adjacent particles lying within a particle's field cone small. That is to say, the granularity of the charge density represented by the macroparticles has to be, depending on

\footnotetext{
* Work supported by DFG under contract No. Ri 242/12-1 and through the Graduiertenkolleg "Physik und Technik von Beschleunigern"

\# Email: staats@temf.tu-darmstadt.de
}

energy, sufficiently fine-grained in order to model the physical interaction properly.

A grid-based algorithm for field calculation determines the space charge fields from a charge density free from granularity, typically modeled linearly between the grid points. The number of particles $N$ required for approximating the charge density appropriately is therefore independent of energy. In addition, assuming the minimum number of particles per grid-cell being fixed, the computation time for determining the fields in a gridbased algorithm is linear in $N$ when using a multigridmethod. The number of operations in point-to-point calculations however is of $O\left(N^{2}\right)$.

\section{GRID BASED METHOD}

An objectoriented 3D tracking program $\mathbf{Q}$ has been developed [2] realizing a grid-based tracking algorithm.

The finite difference Poisson solver developed for the tracking code Q uses a geometrical Full-Multigrid-method [3]. This algorithm's linear dependency of the number of operations on the number of gridpoints is shown in Fig. 2. After the conversion of the charge-density onto a coarsest grid, Poisson's Equation is solved directly. The solution is succesively interpolated onto the next, by a factor of two finer grid, on which high frequency errors are smoothed by several relaxation steps using a classical iteration scheme. Low frequency errors are smoothed by multigridcycles of $\mathrm{V}$ type. The electric field at the distinct particle positions is determined by linear interpolation and transformed into the lab frame (see Fig. 3).

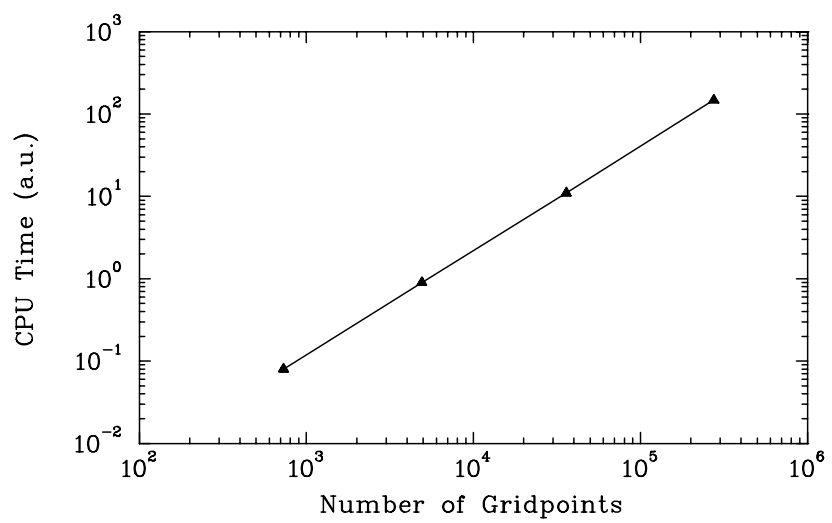

Figure 2: CPU-time for solving Poisson's Equation with a Full-MG solver showing a linear growth. 


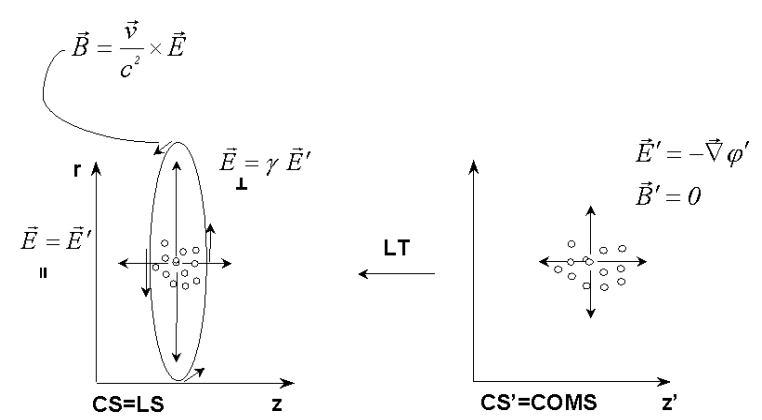

Figure 3: Lorentz-transformation of the electric field.

\section{POINT-TO-POINT METHOD}

The simulations with a code using a point-to-point method to calculate the space charge fields were performed with the General Particle Tracer (GPT) [4], a well established 3D tracking code. As tracking code Q it uses a fifth-order embedded Runge-Kutta method with adaptive stepsize control to integrate the particles' equations of motion.

The space charge induced fields at the position of particle $i$ are the summation of the fields generated by the particles $j \neq i$. In the rest frame of macroparticle $j$ this particle generates an electric field

$$
\vec{E}_{j \rightarrow i}^{\prime}=\frac{q \vec{r}_{j i}^{\prime}}{4 \pi \varepsilon_{0}\left|\vec{r}_{j i}^{\prime}\right|^{3}}
$$

which acts on particle $i, \vec{r}_{j i}^{\prime}$ is the distance measured in the rest frame. Transforming back to the lab frame yields the electromagnetic fields. To avoid unrealisticly strong defocussing effects if two macroparticles are moving close together, the macroparticles act as clouds with radius $R$ instead of pointlike charges. Overlapping of two homogeneous particle clouds results in a reduced electric field:

$$
\vec{E}_{j \rightarrow i}^{\prime}=\frac{q \vec{r}_{j i}^{\prime}}{4 \pi \varepsilon_{0} R^{3}} \text { if }|r|<R
$$

The results of the simulation strongly depend on the number of macroparticles $N$ per bunch. By increasing $N$ the effect of granularity, which induces an overestimation of the space charge effects, is reduced.

For a given number of macroparticles the radius of the particle clouds takes an effect on the results. An oversized radius reduces the space charge fields. By reducing the radius the behaviour of the bunch like the emittance and the transverse standard deviation converges.

\section{COMPARISON CALCULATIONS}

In the model problem a uniformly charged cylindrical beam with a total charge of $1 n \mathrm{C}$, a transverse standard deviation of $\sigma_{\mathrm{x}}=0.5 \mathrm{~mm}$ and zero emittance drifts at an energy of $10 \mathrm{MeV}$ over a length of $1.5 \mathrm{~m}$. These beam parameters are comparable to those being discussed to arise in the capture cavity of the Tesla Test Facility, there with a normalized emittance of $1 \mathrm{~mm}$ mrad .
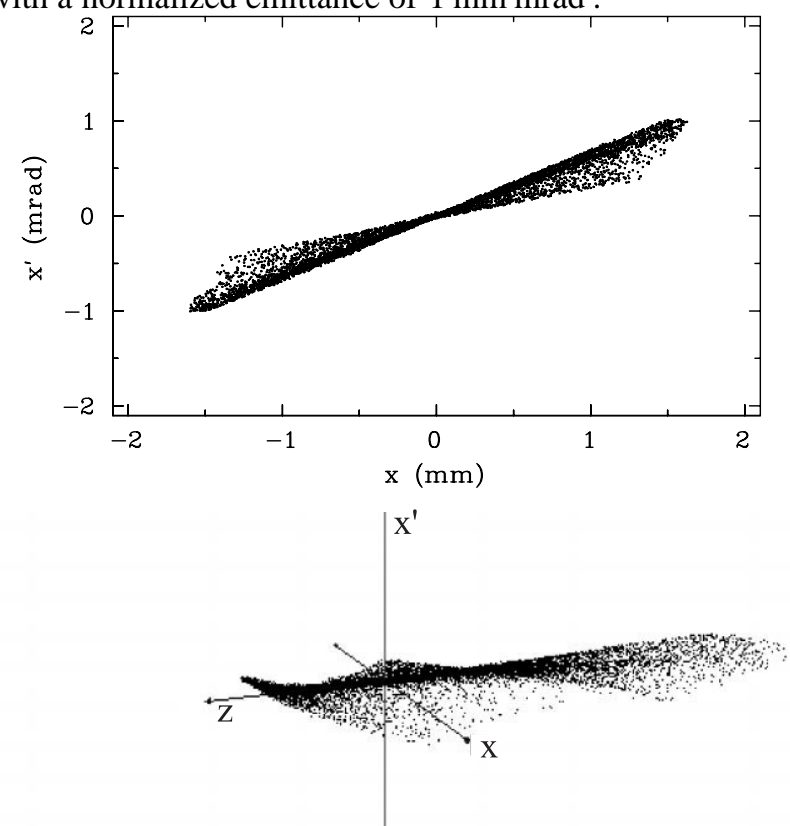

Figure 4: Phase space distribution after $1.0 \mathrm{~m}$ drift calculated with $\mathbf{Q}$. The deviation from a line shape is due to nonlinear transverse effects at the head and the tail of the bunch, as can be seen in the 3D plot with $\mathrm{z}$ (direction of drift) as the second abscissa.

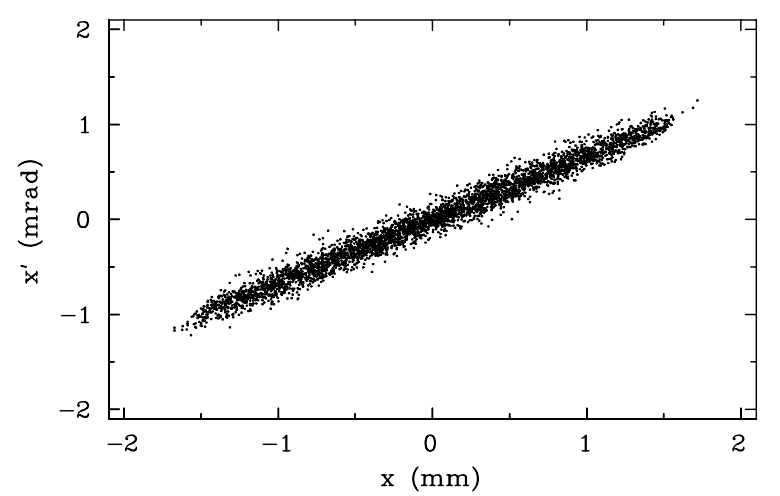

Figure 5: Phase space distribution after $1.0 \mathrm{~m}$ drift calculated with GPT.

There is a good agreement in the results of the spacial standard deviation between the two models (see Fig. 6). In the point-to-point method the description of the emittance as the most significant statistical quantity is rather strong dependent on the number of macroparticles. The low dependency of the emittance determined with the gridbased method is due to the fact, that the charge density used for calculating the electric field is free from granularity.

This independency of the determined space charge forces on statistics gives rise to the description of nonlinear effects away from the beam's longitudinal center and a conservation of phase space volume at fixed $\mathrm{z}$ (see Fig. 4). 
The deviation in the phase space plot calculated with GPT (see Fig. 5) is significant. In the center of a symmetric relativistic beam the determined phase space volume is not conserved due to a high statistical relative error in the relativistic electromagnetic fields determined from point-to-point calculations.
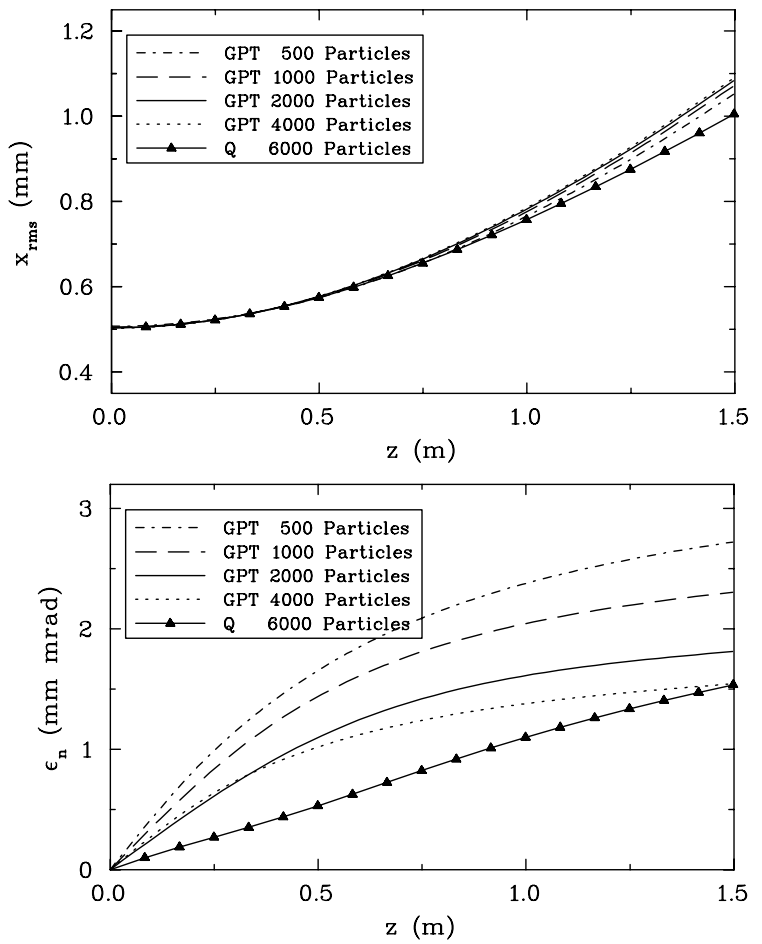

Figure 6: $x_{\mathrm{rms}}$ and transverse emittance along driftspace.

\subsection{Particle-in-Cell Method and analytical linear Model}

In a Particle-in-Cell scheme (eg. the 21/2-dimensional MAFIA TS2 code [5]) the coupled problem of Maxwell's Equations and the equations of motion are solved consistently. As can be seen from calculations with a Gaussian beam $\left(\sigma_{z}=0.8 \mathrm{~mm}\right)$, presented in Fig. 7 , the methods lead to comparable results on the first $0.5 \mathrm{~m}$. The deviation arising in the TS2 result is understandable due

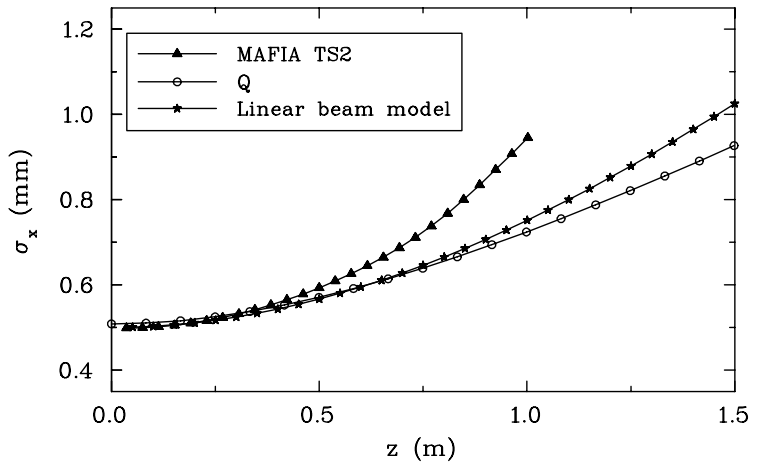

Figure 7: $\sigma_{\mathrm{x}}$ of a Gaussian beam $\left(\sigma_{z}=0.8 \mathrm{~mm}\right.$ at 10 $\mathrm{MeV}$ ) along driftspace. to numerical noise in the field calculation, which would be compensatable by an increase in the number of particles only.

The high number of time steps, which are necessary to dissolve the beam's charge-density spacially (Courant Levy Condition), is countered by the fact, that the problem of field calculation is restricted to a matrix-vector multiplication [5]. The main computation time in an electrostatic code on the other hand is needed for solving Poisson's Equation. It's stability in time depends mainly on the trajectories' integration algorithm. Computation time thus depends on the interval, within which the assumption of a piecewise constant space charge field holds and can remain practible for long distance calculations in such cases.

Alternatively to particle methods the calculation with an analytical model for a self-consistent description of a beam in an accelerator [6], which takes into account linear forces and is suited for rapid estimations of the significant beam parameters, leads to a comparable result.

\section{CONCLUSIONS}

We find agreement between calculations with the tracking algorithm using grid-based space charge fields $\mathbf{Q}$, the point-to-point method GPT and TS2 concerning the spatial distribution. The description in phase space however leads to different results. One explanation favoured by the authors is, that in a point-to-point method the granularity of the charge density modeled by the macroparticles limits the achievable accuracy of the space charge forces determined in a relativistic beam. This leads to a lower estimation of nonlinear effects away from the transversal center of a space charge dominated electron beam and to a stronger statistical error in regions of linear space charge forces. The statistical limits of a Particle-inCell code such as MAFIA TS2 due to numerical noise in the field calculation were shown.

\section{REFERENCES}

[1]J. Staats, "Simulation of Low Energy Electron Beams", Poster in the course of CERN-Accelerator School September'97 in Gjøvik, Norway.

[2]J.Staats, T.Weiland,"Tracking Program Q for the Simulation of Low Energy Electron Beams in a Linear Accelerator", Grako News 1/98, Graduiertenkolleg Physik und Technik von Beschleunigern, (1998)

[3]William H. Press, Brian P. Flannery, Saul A. Teukolsky and William T. Vetterling, "Numerical Recipes, The Art of Scientific Computing", Cambridge University Press, 2nd edition, (1992)

[4]GPT User Manual, Pulsar Physics, Flamingostraat 24, 3582 SX Utrecht, The Netherlands.

[5]P. Schütt, this conference

[6]A. Novokhatski, T.Weiland, "The Model of Ensembles for the Beam Dynamics Simulation", this conference 